\title{
A CASE OF SYSTEMIC BLASTOMYCOSIS
}

\author{
M. LEWISUN, M.D.: AND H. JACKSON, M.D. \\ CHICAGO
}

Case XXI (3).-Wistory-M. C.., Ttalian, aged 17, was admitted to the Cook county llospital, May 20, 1907, to the service of Dr. Goodkind, with the following history: His illness begin five months ago with the appearance on the anterior surface of the middle of the right thigh of an abscess which ruptured and discharged a thick, fetid, dark-brown pus. A papillomatous crust formed subsequently at the site of the abscess. There was at the same time a small abscess near this which healed in a few days. Two months later a sharp stabbing pain occurred in the right knee. Motion soon became restricted and in two weeks a swelling of the joint appeared. This gradually increased so that in two nonths the knce was twice its bormal size. It remained in this condition until the time of his aclmission to the hospital. One month after the onset of the pain in the right knee, the left knee became similarly involved, but the pain and swelling were less severe. The right ankle also became affected, but the process did not progress and at present (the time of his admission) shows no signs of inflammation. Marked swelling, accompanied by pain and tenderness, oceurred successively in the left ankle, both elbows, left wrist and the first metacarpophalangeal joint of the left hand. A small papule developed in the left eyebrow one month after the commencement of the joint troubles, and since then similar Jesions have appeared on other parts of the face and head. (Fig. 1.)

The patient was born in Italy and from the age of 10 was engaged in hard manual labor on a farm. Four years ago he came to the United States and las worked at intervals as an organ-grinder and helper in a machine shop, until the onset of the present illness. He lived in the congested ltalian district on the west side of ('hicago, and his living quarters were unhygienic in the extreme. The rooms themselves were dry at the time of investigation (summer) but the kasement in which the closets were located was wet, and decomposing feces and wool were found in many places. His health had always been good and he denied venereal infection. The family history was unimportant except that his father died two years ago after an illness of two nonths during which time he coughed frequently, often expectorating blood.

First Admittance to Mospital.- When admitted to the hospital, on accoum of the multiple arthritis, the diagnosis of ehronic rheumatism was mate. 'The course of tlie disease, however, during the subsequent two months, led to the consideration of tubereulosis and syphilis as possible explanations of the symptoms, and the latter was favored because of an interpretation given to the appearance of the bones of a roentgenogram of the hand. The subsequent development of an abscess beneath the scalp, and its characteristics resembling those encountered in other cases of blastomyeosis, led to the examination of aspirated pus and the discovery of the organisms. The patient now was anemic and emaciated; seemed quite comfortable and complained of pain and stiffness: of the joints. The pulse was 108 ; temperature $101.8 \mathrm{~F}$.; respirations 28 . On the face were a number of small oval, indurated, purplish ulcers, their edges leing fairly regular and rounded margins sloping outward to the skin around them. The largest of these situated above the right eye and measured 6 by 20 $\mathrm{mm}$. Other similar lesions in various stages of involution were present on the back of the neck, scalp, arms, thighs and left ankle. An oval "rice-paper" scal" was present on the upper part of the right thigh, and below this a fistulous opening discharged bloody pus. It was surrounded by an oval area of indolent 
granulation tissue and represented the site of rupture of the first abscess which appeared. Beneath the scalp three small abscesses were found; one of these was more deeply situated and seemed to be connected with the bone.

The eyes, ears, nose and neck were normal. The chest was negative on inspection except over the tenth rib in the posterior axillary line on the left side, where tilıre was a soft rounded swelling the size of a hazelnut, and evidently attached to the rib. Resonance was impaired over both apices in front and

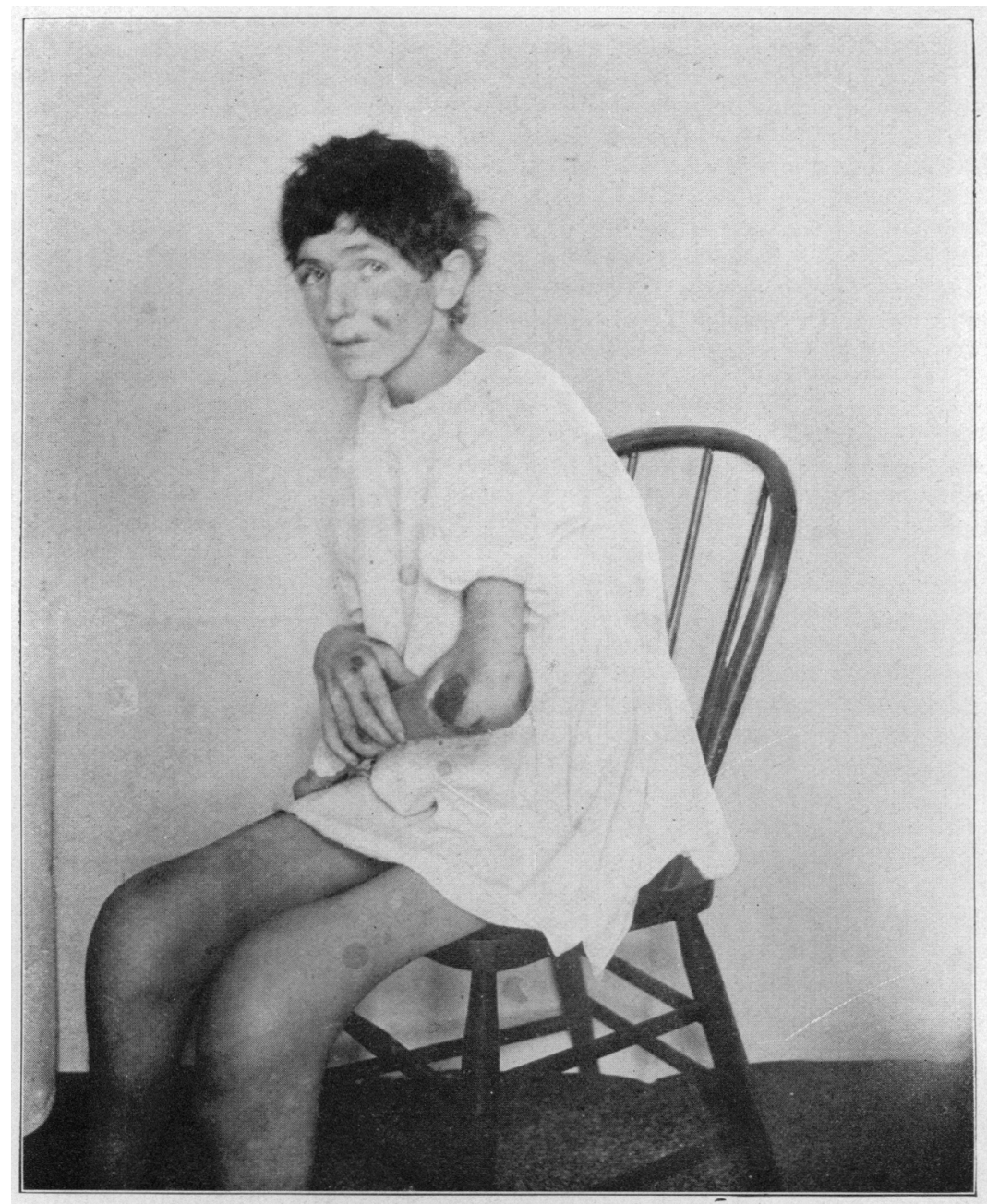

Fig. 1.-M. C., photographed a few days before death. Note the cutaneous lesions on the left cheek and right hand, the swollen and ulcerated condition of the left elbow, and the marked swelling of the knees.

behind. The respiratory murmur was roughened and expiration prolonged. No râles were heard anywhere. Posteriorly below the left scapula, resonance was impaired and the voice and breath-sounds slightly diminished. A faint systolic murmur over the pulmonic area and a slight enlargement of the liver were the only alterations in the further examination of the chest, abdomen and genitalia. 
The muscles of the extremities were atrophied. Both elbows, the right wrist, both knees and the left ankle were swollen and tender, and motion was restricted and painful. There were in the right hand two small abscesses involving the first metacarpophalangeal joint and the middle phalanx of the fourth finger. A sinus on the palmar surface of the fourth finger communicated with the lastnamed abscess and disenarged a thick brown pus. The left wrist was tender, and extending from an abscess in the first metacarpophalangeal joint were two sinuses, one dorsal and one palmar, each discharging a thick yellow pus.

There were no disturbances referable to the nervous system. The superficial cervical and epitrochlear lymph-nodes were slightly enlarged.

The urine was amber, clear, acid in reaction, specific gravity 1.018, and contained no albumin. sugar or casts. No blastomycetes were found in the urine obtained after massage of the prostate. Examination of the feces failed to show anything of importance.

The blood contained $3,800,000$ red cells per cubic millimeter; 9,600 white cells and 65 per cent. hemoglobin (von Fleischll). A stained specimen showed slight poikilocytosis but no nucleated red eells. The differential white count was as follows: polymorphonuclear neutrophils, 55 per cent.; small lymphocytes 32 per cent.; large tymphocrtes, 11 per cent.; eosinophils 2 per cent. To cultures were obtained from the blood in several attempts.

The course of the disease was slow and marked by several remissions and exacerbations. The pulse ranged from 80 to 120 ; the temperature was highest in the evening and varied between 98 and $103.6 \mathrm{~F}$. After June 22, when the diagnosis was established, the patient was given increasing doses of potassium iodid, tonics and fresh air treatment and rapidly improved. The pain in the joints disappeared and he left the hospital July 8 and resumed his occupation, organgrinder.

Second Admittance to Hospital.-Ten days later he returned to the hospital with a marked aggravation of all his joint-symptoms. He coughed much, expectorating slight amounts of mucnid material, and suffered greatly from pain in the joints; the pulse was 84 ; temperature 100.8 ; respirations 24 . The right ankle, which was normal previously, was swollen and tender, and two new abscesses had appeared on the ulnar side of the right wrist and on the tarsal surface of the left wrist extending into the land.

The physical signs in the lungs were also more pronounced, but no râles were heard. Administration of potassium iodid was resumed with no improvement for about eight days. Many new abscesses appeared successively on the hands and feet, and the temperature ranged up to $103.4 \mathrm{~F}$. Copper sulphate was now given internally in $1 / 4$ grain doses three times a day and the hands and feet, in which were many discharging abscesses, were dressed with hot 0.5 per cent. solution of copper sulphate. The patient again improved, the congh subsided; the abscesses healed and the joints became less painful.

Repeated examinations of the sputum were made for the tubercle bacillus and blastomycetes with negative results until August 5, when budding blastomycetes were found. The improvement continued and the patient gained in weight and strength. The temperature did not rise above $99 \mathrm{~F}$. after August 11. All the fistulas closed and he left the hospital August 17 in good condition, there being only slight limitation of motion in the elbows and knees. At the time of discharge 150 grains of potassium iodid three times a day and $1 / 2$ grain of copper sulphate three times a day were being given.

Third Admittance to Hospital.-The patient again applied for admission to the hospital Dec. 12, 1907, and was assigned to the service of Dr. Mix and afterward transferred to the surgical service of Dr. Ryerson. During the second furlough of four months he suffered many hardships. He had taken no medicine, and had tried to return to Italy, but the officials at New York refused him passage and he was forced to sell papers on the streets in order to obtain food. He entered the hospital much emaciated and in a very weak condition, 
with an exacerbation of all his old lesions and some new ones. The pulse ranged from 90 to 130 , temperature 98.6 to $103.6 \mathrm{~F}$., respirations 22 to 30 . There was a general adenopathy, especially noticeable in the axillary, cervical and inguinal regions. Large abscesses involving both knees, wrists, elbows and ankles were present, the one about the right knee being very large and fluctuating and extending half way up the thigh. The others had ruptured and discharged a thick yellow pus. He suffered excruciating pain on movement of any of these joints. The ulcers of the skin were larger, and new lesions were present on the lip and the back of the neck. Impaired resonance and harsh breathing obtained over the apices of both lungs.

Increasing doses of potassium iodid and a nutritious diet were again administered, but the patient became weaker despite treatment and died Jan. 16, 1908.

Early in the course of the disease, Bier's passive congestion treatment was useri, but it seemed to do little good. As soon as the patient was able to leave his bed he spent nine hours a day in the sunshine and open air.

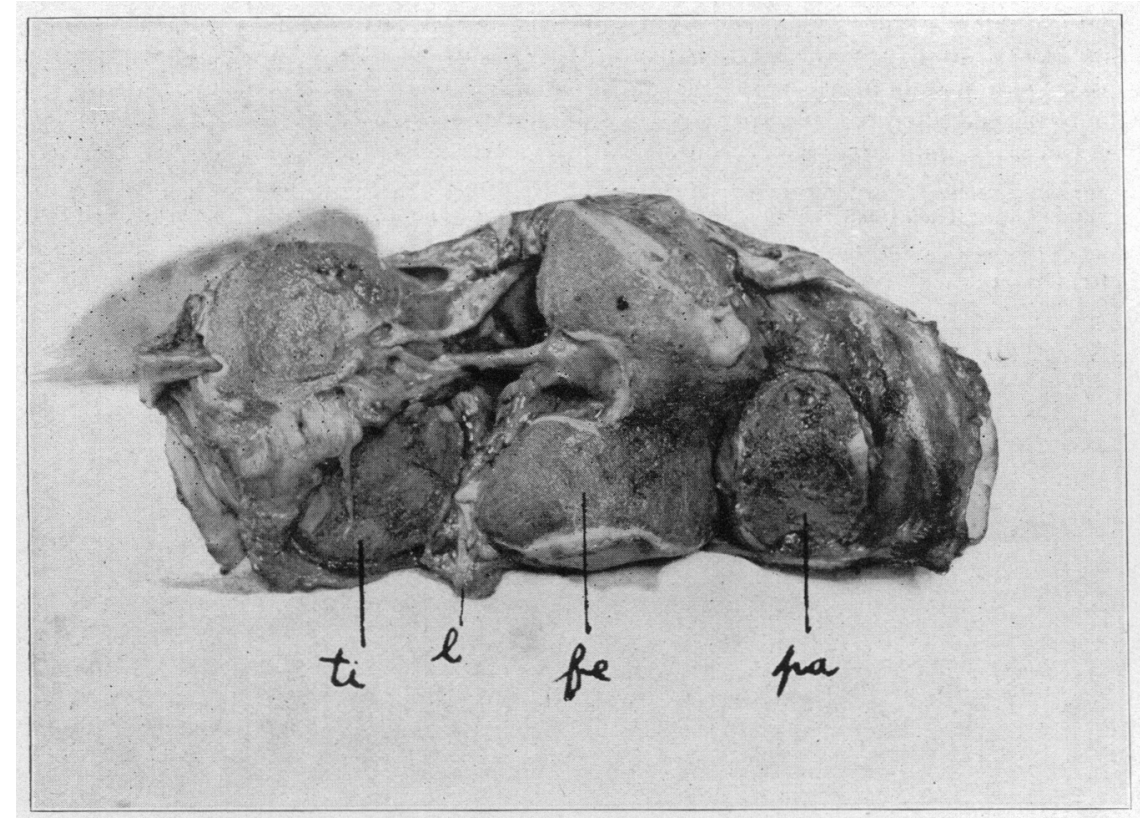

Fig. 2.-Articular surfaces of left knee: $t i$, eroded surface of head of tibia; fe, lower end of femur on which only a portion of the articular cartilage remains: pa, patella, in which are two abscesses, the openings of which are exposed; $l$, articular ligaments which were so disorganized that subluxation occurred.

Necropsy.-A post-mortem examination (Dr. Stober) was held two days after death and the following is the anatomical diagnosis: indurated blastomycotic bronchopneumonia of the left lung; blastomycosis of bones, subcutaneous and cutaneous tisues, pons Varioli, inguinal and axillary Jymph-nodes; acute vegetative nitral endocarditis; fatty liver; parenchymatous degeneration of the kidners; fibrous pleuritis; hyperplasia of the lymphoid tissue of the ileum; edema of the lungs; hydropericardium; atrophy of the heart, pancreas and testicles; emaciation and anemia.

The body is that of an extremely emaciated young man. Numerous cutaneous lesions, abscesses and fistulas are present, the majority of which have been described in the clinical report. Both knees are narkedly swollen and the 
skin covering them contains several ulcers and discharging fistulas. A posterior subluxation has occurred in the right knee, and the same condition can be produced in the left knee. The joint-cavities contain a large amount of bloody pus, the periarticular tissues are disorganized, the ligaments destroyed and the cartilages have disappeared, exposing the rough granular surface of the bones (Fig. 2.) About these joints large communicating abscesses exist; the one above the left knee extends the whole length of the thigh and contains nearly a quart of pus. Another above the right knee is about half as large. Discharging abscesses involving the elbows and the small bones of the feet are also present. 'The articular structures of the latter have been practically destroyed. There are abscesses conmunicating with the lymph-nodes in both groins and in the right axilla. The lympl-nodes of the left axilla are enlarged and pink on section.

The pleural cavities contain about 2 ounces of a clear yellow fluid, with slight fibrous adhesions about the apices and also to the diaphragm on the left side. The pericardial cavity contains 4 ounces of a clear yellow fluid.

The lungs weigh $620 \mathrm{gm}$. Both crepitate freely and do not collapse. The left lung on section is mottled light gray and on pressure exudes a slight amount of frothy fluid. In the extreme lower margin of the lower Iobe and externally is a region of induration roughly $5 \mathrm{by} 1 \mathrm{~cm}$. in diameter, somewhat circular in outline and cliveted toward the root of the lung, where it is continuous with a similarly indurated tissue behind the main bronchus, in which there are several yellow regions of newosis (Fig. 3). The pleurae overlying the consolidated lung tisine next the diaphraym is thickened and binds the lung to that structure. The lung beneatl contain: mumerous small blood-vessels and bronchi surrounded by firm tissue heavily pigmented with conl-dust. The pericardium is generally adherent to the left lung. The remainder of this lung as well as the right lung is unchanged. The tracheo-bronchial lymph-noles contain large amounts of coal pigment and are slightly enlarged.

The tongue is normal. The tonsils are small and from their crypts a purulent material is expressed. Tlee esophagus is smooth; the trachea and larynx are slightly lyperemie and contain mucopus.

The heart weighs $240 \mathrm{gm}$. The pulmonary tricuspid and aortic valves are normal. The mitral valve is thickened and on the edge of both cusps are fine waty vegetations the largest of which measures $1 \mathrm{~mm}$. in height. The heartmuscle is pale; the left ventricle wall measures $5 \mathrm{~mm}$. in thickness, the right $2 \mathrm{~mm}$. The arta and coronary vessels are normal. The liver weighs 1,400 $\mathrm{gm}$. Its surface shows the imprint of the ribs. On section the tissue appears somewhat opaque and light yellow. The spleen weighs $200 \mathrm{gm}$. Its capsule is thickened, its consistency firm, the cut section brown, and there is an increase in the connective tissue. No mieroscopic nodules are present.

The brain weighs $1.600 \mathrm{gm}$. It is normal externally. In numerous sections. one b'astomycotic nodule is found in the pons a few millimeters below the ventral surface.

The kidneys weigh $280 \mathrm{gm}$. On section the kidney substance bulges; it is a grayish-yellow; the cortex is increased in thickness and the capsule strips readily, leaving a smootlı surface.

The testicles are small, and sections expose an increase in connective tissue. The bladder is small and contains a small amount of turbid urine. The rugae are fairly prominent; the posterior urethra is congested and slightly granular. The prostate and seminal vesicles are nornal. The pancreas is firm and small. The mucous membrane of the stomach is thin, and small ecchymoses are present about the pyloric region. The intestines are normal.

The left leg was amputated through the thigh for a more detailed examination. A small opening into the knee-joint from an abscess in the internal condyle was found, as well as several small regions of softening in the cancellous bone of the patella. The abscess in the condyle was $3 \mathrm{~cm}$. in diameter and the passage into the joint one-third as large. The bone about the 
abscess as well as that just beneath the articular cartilages of both tibia and femur was soft and diffusely yellow; on crushing a semipurulent fluid was obtained (Fig. 4). On the antero-internal surface of the tibia at the junction of the upper and middle thirds an opening $5 \mathrm{~mm}$. in diameter was found passing through a raised bony and shell-like periosteum under which was a small aliscess not connerted with the marrow carity. 'The epiphyses of the Iower end

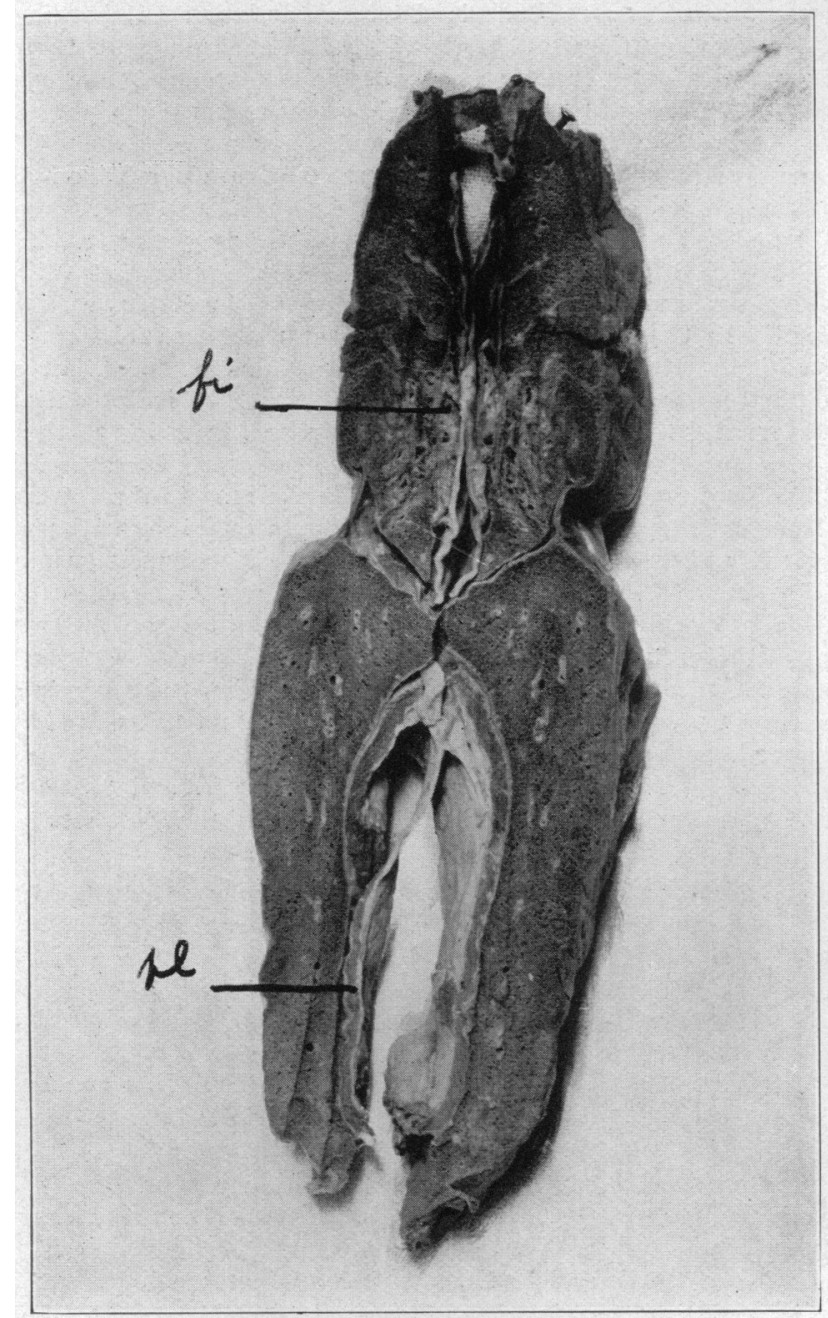

Fig. 3.-Section through the lower lobe of left lung: $f$, fibroid area representing healing blastomycotic lesions; $p l$, thickened pleura adherent to the diaphragm.

of the tibia and fibula also contained small yellow softened regions communieating with abscesses over both malleoli which had ruptured externally (Fig. 5), the one over the external malleolus continuous with an abscess extending beneath the Achilles tendon. Occupying the greater part of the dorsum of the foot was a sharply demarcated region of necrosis, and the tissues bordering this were 


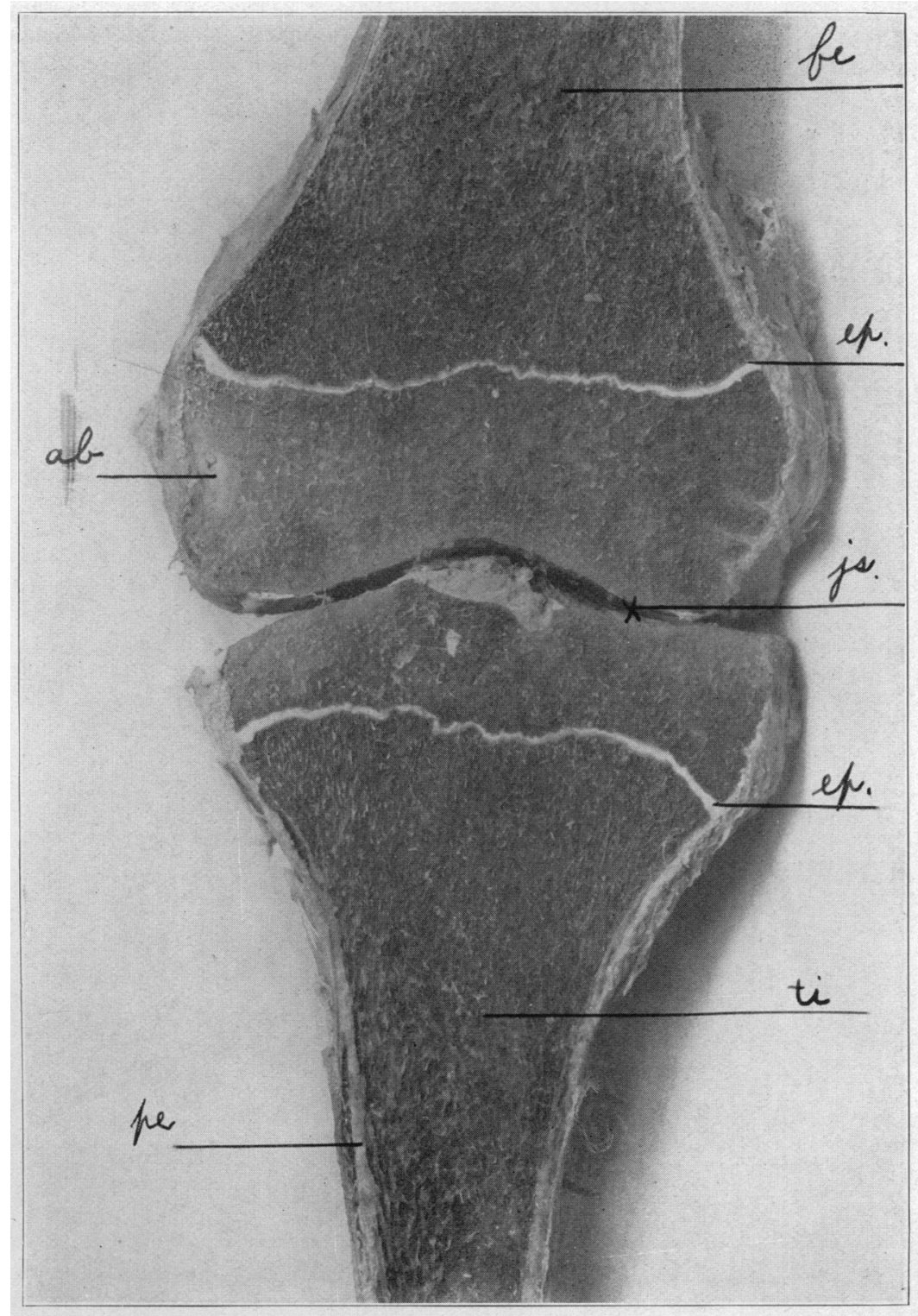

Fig. 4.-A longitudinal section of the left knes-joint: fe, lower end of femur; $t i$, upper end of tibia; ep, epiylyseal line; $j s$. roded joint surfaces; the light color of the adjacent bone represents the extent of the blastomyentic process; pe, healed periostitis; $a b$, abscess in epiphrsis which communicated with the joint. 
without evidence of reaction. In this region the unchanged extensor tendons lay bare, and beneath were the fragments resulting from the disorganization of several of the tarsal and portions of the metatarsal bones (Fig. 6). In the pus of the joint cavities, bone abscesses and adjacent soft cancellous marrow were to be found many round and budding blastomycetes, some of which possessed capsules of unusual thickness.

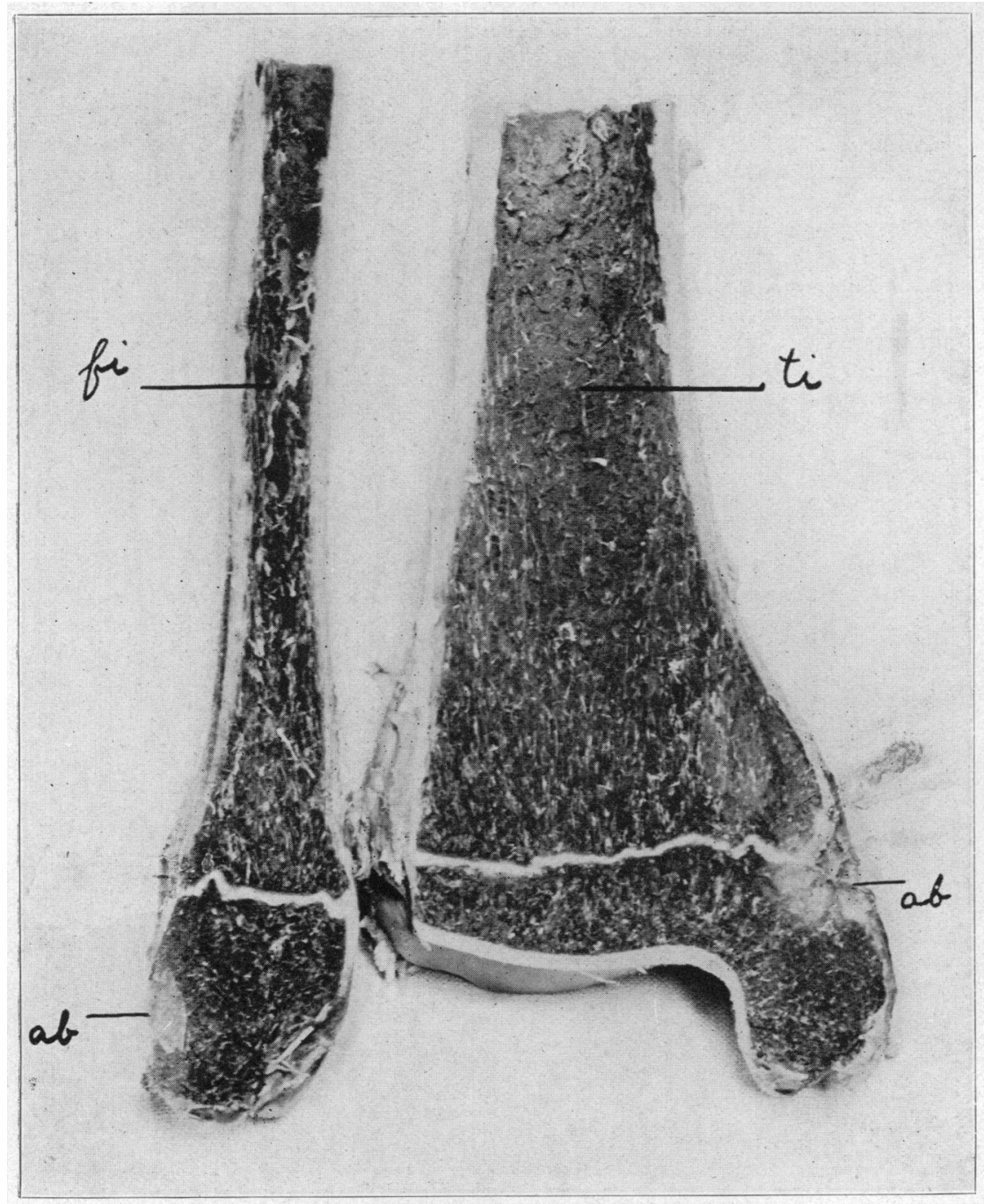

Fig. 5.-Longitudinal section through tibia $(t i)$ and fibula $(f i)$ with abscesses $(a b)$ in the epiphyses.

Histology.-In sections of the indurated region in the left lung, compressed and distorted bronchi were found surrounded by masses of adventitious fibrous tissue; one of these bronchi contained enormous numbers of blastomycetes. Several of the minute so-called abscesses with organisms and the other features of such blastomycotic local lesions were scattered about in this fibrous tissue. The 
other alterations were those commonly encountered about regions of chronic disease in the lungs.

The changes in the bones were of special interest on account of the extensive necrosis. Sections were studied from the abscesses in the patella, fibula, femur and tibia, and the alterations were quite similar. The centers of small abscesses and the tissue near the surfaces of such as had discharged their contents were made up of disorganized and necrotic bone, leukocytes and red cells. In the sections from the yellow and soft cancellous bone beneath the articular cartilages

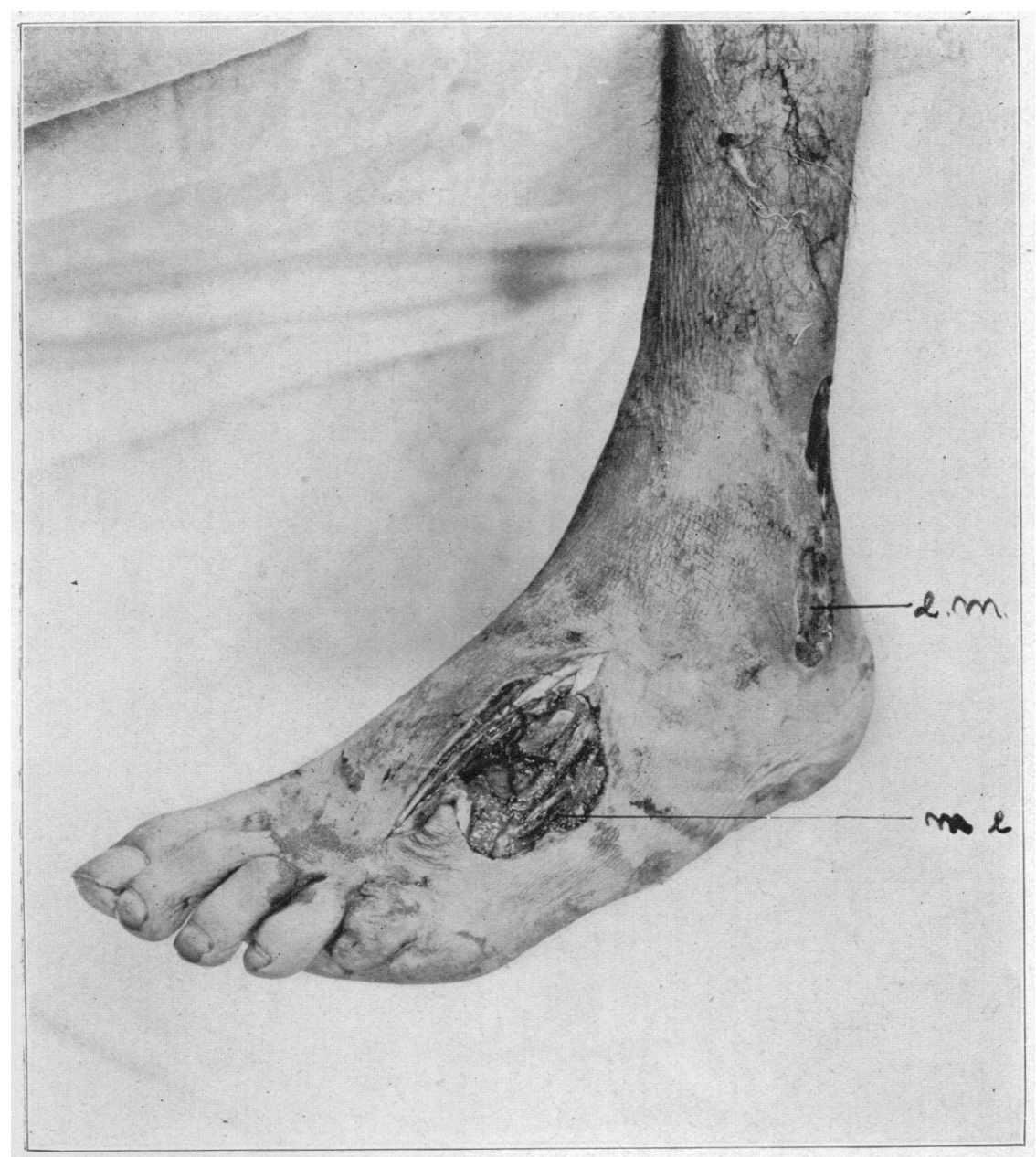

Fig. 6.-Ulcerations communicating with blastomycotic abscesses in the metatarsus $(m e)$ and external malleolus $(\mathrm{em})$. Muscles, bones and tendons have been exposed.

there was a diffuse necrosis for the depth indicated in the gross description, a necrosis evidently due to the rather regular extension into the head of the bone of the blastomycosis of the joint. This diffuse necrosis was difficult to explain by the coalescence of local lesions. In some sections zones of mononuclear leukocytes surrounded the necrotic tissue, but no evidences of granulation tissue about 
these was found, and organisms occurred outside the lesions in the cavities of the marrow where but little change had taken place. Histological changes in accord with the gross description. both indicating the formation of new bone from the cambium, were found in the shell-like but perforated and imperfect outer wall of the abscess on the inner surface of the upper part of the tibia.

In the inguinal lymph-nodes not only were focal lesions present, such as have been repeatedly described in the tracheobronchial lymph-nodes, but also peripheral lymph sinuses were found crowded with organisms, evidently in the current since the adjacent tissues were without alterations. In the spleen, too, organisms were found; at one point a clump of fifteen or twenty, at another, budding forms and about them no evidences of tissue reaction. The lesion of the pons, $3 \mathrm{~mm}$. from the ventral surface and approximately $1.5 \mathrm{~mm}$. in diameter, contained numerous organisms and was apparently formed by the coalescence of more minute local lesions. The changes in sections of other tissues and organs conformed to the anatomic diagnosis.

Bacteriology.-The Staphylococcus albus and aurcus were recovered from the blood, pleural fluid, bile and pus from the abscesses of the knee-joints, left inguinal and right axillary regions. Blastonycetes were demonstrated in the pus of the abscesses mentioned as well as in the necrotic material from numerous bone lesions, but were not obtained in culture. The failure to cultivate these organisms from material yielding cultures during life is not an infrequent postmortem oceurrence.

This report is of more than ordinary interest on account of the improvement which occurred during the first two periods in the hospital, and also because of the existence of relatively slight blastomycosis in the lungs at the time of death - the organisms having disappeared from the sputum some time previously and coincidentally with the fewer evidences of pulmonary alteration on physical examination - and the very extensive involvement of the subcutaneous tissues, bones and joints. 